zieht sich wie ein roter Faden durch das Buch Laternsers. Der Anwalt bemüht sich, Anklagepunkte zu entkräften, Kompetenzen zu ordnen und zu begrenzen, so schauerlichen Erscheinungen wie den >Einsatzgruppen des SD ihren richtigen Platz innerhalb des Cefüges der verschiedenen Befehlsbereiche zuzuweisen. Er überzeugt oft, jedoch nicht immer. Die ärgste Schwierigkeit bei der Erörterung all dieser Dinge liegt im Bereich des Rechts selbst, liegt in dem, was englische Völkerrechtler, welche Laternser freigebig zitiert, als die $>$ Unbestimmtheit des Kriegsrechtes $<$ bezeichnet haben. ${ }^{163}$

Darüber hinaus ging Görlitz neben den angedeuteten Aspekten des internationalen Kriegs- und Völkerrechtes ${ }^{164}$ sowie der (individuellen) »Schuldfrage « zweimal auf die Einsatzgruppen ein. Zum einen beim »Fall Kesselring«. Hier schrieb Görlitz:

Kesselring etwa hatte, im Gegensatz zu den Heeresgruppenbefehlshabern in Rußland, in Italien auch die Befugnis eines Territorialbefehlshabers. Mußte er sich in schwierigster militärischer Lage auflehnen, als die Liquidierung von Hunderten von Ceiseln dem ihm nicht unterstehenden SD überantwortet wurde? Die Antwort kann kein irdisches Cericht entscheiden, sie steht dem Menschen $\mathrm{zu}^{165}$

Zum anderen ging er im Zusammenhang mit der »deutschen militärischen Résistance« auf die Einsatzgruppen ein. Hier hieß es bei Görlitz: »Es sollte nachdenklich stimmen, wenn ausgerechnet Führer von SD-Einsatzgruppen, Massenmörder vom Schlage eines Ohlendorf, die alte Offiziersgeneration anklagen, in der sie ihre alten Gegner erblicken. «"166

\title{
1.7 Die Memoirenliteratur und der deutsche Widerstand gegen den Nationalsozialismus oder: das "Erinnerungsbuch " Ernst von Weizsäckers
}

Im Zusammenhang mit der Auseinandersetzung der Memoirenliteratur soll im Folgenden ausschließlich auf den Beitrag Die verhinderten Hochverräter. Wege und Irrwege neuerer deutscher Memoirenliteratur von de Mendelssohn ${ }^{167}$ eingegangen werden. Dies erfolgt aus mehreren Gründen. Die Darstellung verkörperte - im Vergleich zu den bereits genannten Artikeln sowie weiteren Beiträgen zur Memoirenliteratur im Mo-

163 Ebd., S. 431.

164 Ich komme auf diesen Punkt im Schlussteil eingehend zu sprechen.

165 Ebd., S. 431.

166 Ebd., S. 432. Vgl. auch die Ausführungen über Otto Ohlendorf und über seine Rolle in der SS und im RSHA, die sich nach Gerhard Ritter »zu einer Art von Zentralbehörde des `Himmler-Staates« entwickelte - ohne indes auf die spezielle Bedeutung und Aufgaben der Einsatzgruppen im Kontext des Zweiten Weltkrieges einzugehen und ohne die Rolle Ohlendorfs als Leiter der »Einsatzgruppe D«zu beleuchten: Gerhard Ritter, Carl Coerdeler und das Ende des deutschen Widerstandes, in: Der Monat 7 (1955), H. 77, S. 387-401, hier S. 392 f.

167 Peter de Mendelssohn, Die verhinderten Hochverräter. Wege und Irrwege neuerer deutscher Memoirenliteratur, in: Der Monat 3 (1951), H. 29, S. 495-509 ([Sammel-]Rezension). Vgl. auch ders., Kritiker auf schlechtem Sitzplatz, in: Der Monat 4 (1952), H. 41, S. 544-546 (hierbei handelte es sich um die Besprechung des Buches: Dr. Paul Schmidt, Der Statist auf der Galerie 1945 bis 1950/Erlebnisse, Kommentare, Vergleiche, Bonn o. J). 
nat ${ }^{168}$ - die substanziellste Veröffentlichung zu diesem Thema: sowohl was den quantitativen Aspekt (15 Seiten) anbelangt als auch aufgrund der hier erfolgten Beschreibung und Analyse des deutschen Widerstandes gegen den Nationalsozialismus, genauer des Widerstandes des "20. Juli«; zudem stellte der Beitrag im Vergleich zu den weiteren Veröffentlichungen $\mathrm{zu}$ diesem Thema ${ }^{169}$ die "schärfste« Auseinandersetzung dar. ${ }^{170}$ Dies schlug sich auch in einigen Reaktionen der Leser des Monat nieder. ${ }^{171}$ Die

168 Siehe Hans Schwab-Felisch, Die Literatur der Obergefreiten. Neue deutsche Kriegsromane und Kriegstagebücher, in: Der Monat 4 (1952), H. 42, S. 644-651 ([Sammel-]Rezension). Siehe auch F[ritz] R[ené] Allemann, Die Rußlandpolitik des Dritten Reiches, in: Der Monat 3 (1951), H. 32, S. 199-202 (Rezension) sowie Alfred Kellner, Im Schatten der Macht. Zu den Memoiren eines SS-Generals, in: Der Monat 12 (1959), H. 133, S. 79-83 (Rezension).

169 Siehe zum Themenkomplex deutscher (christlicher) Widerstand gegen das »Hitler-Regime«im Einzelnen: M[elvin] ]. L[asky], v. Hassell und die Fäulnis im Staate, in: Der Monat 1 (1948), H. 3, S. 99-102 (Rezension); Hugh R. Trevor-Roper, Die Generäle verteidigen sich, in: Der Monat 2 (1949), H. 14, S. 210-213 (Rezension); ders., Rommels Rolle als Verschwörer, in: Der Monat 2 (1950), H. 17, S. 548550; ders., Der rätselvolle Abwehr-Chef, in: Der Monat 2 (1950), H. 21, S. 317-323 (Rezension.); Helmut Uhlig, Zeugnis des Widerstands, in: Der Monat 5 (1952), H. 51, S. 330-332 (Rezension); Walther Hofer, Geschichtsschreibung im Vakuum, in: Der Monat 5 (1953), H. 58, S. 413-416 (Rezension); ders., Der 20. Juli. Über einige Neuerscheinungen, in: Der Monat 6 (1954), H. 70, S. 401-404 (Sammelrezension); Gerhard Ritter, Carl Goerdeler und das Ende des deutschen Widerstandes, in: Der Monat 7 (1955), H. 77, S. 387-401; Franz Böhm, Widerstandsbewegung oder Revolution? Zur Auseinandersetzung um Carl Coerdelers Kampf gegen Hitler, in: Der Monat 7 (1955), H. 81, S. 220-228; Ferdinand Friedensburg, Memento des Widerstandes, in: Der Monat 9 (1957), H. 102, S. 82 f. (Rezension). Vgl. auch Rolf Schroers, Original-Aufnahmen. Über »Das war unser Rommel « und »Beiderseits der Rollbahn«, in: Der Monat 5 (1953), H. 61, S. 83-86; Der Kampf gegen die Kirche. Aus unveröffentlichten Tagebüchern Alfred Rosenbergs (mit einleitenden Worten von Robert M. W. Kempner), in: Der Monat 1 (1949), H. 10, S. 26-38. Vgl. zum Thema Widerstand des »20. Juli 1944« auch den Beitrag Rüdiger Proske, Brief aus Braunschweig: Prozeß um den 20. Juli. Die Braunschweiger Verhandlungen gegen Otto Ernst Remer, in: Der Monat 4 (1952), H. 43, S. 16-21.

170 Mit folgenden einführenden Worten veröffentlichte die Redaktion des Monat den Beitrag de Mendelssohns, Die verhinderten Hochverräter, S. 495: »Die scharfe Auseinandersetzung Peter de Mendelssohns mit einigen Quellschriften zur jüngsten deutschen Vergangenheit findet nicht in allen Teilen die Zustimmung der Redaktion, so in der Frage des >Unconditional Surrender und in der Gesamtbeurteilung eines deutschen Widerstandes. Wir stellen diesen Aufsatz jedoch zur Debatte und sind bereit, nicht nur den Widerspruch der hier unmittelbar Angegriffenen, sondern jeder Leserzuschrift Raum zu geben, die der Diskussion über die hier aufgeworfenen Fragen etwas Wesentliches hinzuzufügen hat«.

171 In einem Leserbrief antwortete Ernest ]. Salter unter der Überschrift Hätten wir alle auswandern sollen? (Der Monat 3 [1951], H. 30, S. 664 f.) in scharfer Form auf den Beitrag von de Mendelssohn. Nachdem Salter konzediert hatte, dass die einzelnen Fakten »isoliert betrachtet« durchaus richtig wären und in de Mendelssohns Aufsatz u. a. ein »Element des Hochmutes« enthalten wäre, kam er abschließend zu folgender Einschätzung: »Ob man will oder nicht: Mendelssohns Anschauung läuft praktisch darauf hinaus, daß alle Deutschen hätten auswandern oder mit dem Revolver in der Hand sozusagen im permanenten Attentat gegen alle Machthaber leben müssen. Das wird einem sofort klar, wenn man den Maßstab Mendelssohns an die Sowjetunion und die östlichen Satelliten in ihrer heutigen Situation anlegt. Es würde nichts übrig bleiben, als das gesamte russische Volk und mit ihm die anderen Völker als kollektivschuldig zu bezeichnen. Bei einer solchen Parallele könnte man die Weizsäcker und Kordt zu Dutzenden einzeln bezeichnen. Die Memoirenwerke der aus einem der beiden Systeme Geretteten, der Überlebenden und Widerstrebenden weisen mutatis mutandis viele gemeinsame Züge auf. Meiner Meinung nach sind Memoiren wie gerade die Kordts von unge- 
Veröffentlichung von de Mendelssohn steht hier also pars pro toto für weitere Beiträge zum Themenkomplex Memoirenliteratur und deutscher Widerstand gegen das "Hitler-Regime«.

Vor dem Hintergrund seiner einleitenden Worte, dass aus seiner Sicht die »Persönlichkeiten der Schreibenden interessieren « und weniger der tatsächliche »Informationsgehalt des Mitgeteilten, und das in unserer Zeit, in der fast jede politische Entscheidung automatisch auch eine moralische ist «, schrieb de Mendelssohn in Heft 29 des Monat in der Februarausgabe des Jahres 1951 in Bezug auf die "Empfindungen« der »historischen Persönlichkeiten« in der von ihm in seiner Rezension untersuchten »Erinnerungswerke«:

Im Bereich der neueren deutschen politischen Memoirenliteratur wird nun eines sogleich deutlich. Es gibt keinen neuralgischen Punkt in der gesamten Ceschichte der Hitler-Zeit, der diese lebendigen Empfindungen der beteiligten Zeitgenossen stärker hat ausschlagen lassen als der Attentatsversuch vom 20. Juli und die aus ihm folgenden Prozesse und Hinrichtungen.

Und der Autor schrieb an diesem Punkt, mit dem Fokus auf den »20. Juli« gerichtet, folgendermaßen weiter:

Dieses Ereignis hat sich als der Kreuzweg der politischen Moralität etabliert; es vibriert in der ganzen Literatur nach. [...]: so grau, schleppend und lustlos-leblos ihre Darstellung häufig sich dahinbewegen mag - an diesem Punkt zuckt sie unweigerlich schmerzhaft zusammen, erhöht sich der Ton, beschleunigt sich der Atem. Hier reagiert der lebendige Nerv durch alle Betäubungsmittel hindurch. Auch der deutsche Vizeadmiral Kurt Aßmann hält den kühlen und sachlichen Ton seiner Darstellung der Kriegsereignisse, an deren abstandsvoller Objektivität nichts zu bemängeln ist, mit großer Beherrschung bis zu dem Augenblick durch, da es vom 20. Juli zu sprechen gilt. Da taucht plötzlich in der Diskussion der moralischen Zulässigkeit von Hoch- und Landesverrat der Satz auf: >Ein deutscher General tut das nicht: das nicht! «Und man meint, in Tirpitzens Memoiren

wöhnlicher Bedeutung, weil sie Zehntausenden von Leser zeigen, wie es tatsächlich im Innern dieser Systeme aussieht. Menschen, die selbst unmittelbare Erfahrungen und tägliche Berührungen mit den Systemen der modernen Diktatoren gehabt haben oder noch haben, vermögen sich leicht die spezifische psychologische Situation zu erklären, die sich bei dem nach den Konventionen des 19. und selbst 20. Jahrhunderts gebildeten und lebenden Menschen inmitten der Katastrophe zeigen mußte. Die bisherigen Erfahrungen erlauben es nicht, bei der Betrachtung solcher katastrophaler Bruchstellen von Cesellschaft und Geschichte, wie es Nationalsozialismus und Bolschewismus sind, den Maßstab des Eiferers anzulegen und angesichts des komplizierten Prozesses der barbarischen Transformation Mitteleuropas unter dem Nationalsozialismus einfach die Position der üblichen demokratischen Moral zu beziehen." Siehe auch die folgenden Leserbriefe zu de Mendelssohns Veröffentlichung Die verhinderten Hochverräter von Horst Reh, Walter Tschuppik, Stephan von Hartenstein, Dr. E. Schwarz sowie Hermann Glaser, in: Der Monat 3 (1951), H. 31, S. 102-106; unter der Überschrift Noch einmal >Die verhinderten Hochverräter die Zuschriften von Kurt Assmann und Friedrich Carl Westphal (Der Monat 3 [1951], H. 32, S. 215-218); unter der Überschrift Das Geschichtsbild eines Unbelehrbaren die Zuschrift von ak (d. i. Alfred Kellner) (Der Monat 3 [1951], H. 33, S. 328-330); unter der Überschrift Memoiren-Literatur die Zuschrift von T. R. (Der Monat 3 [1951], H. 35, S. 553 f.); unter der Überschrift Ist Aßmann unbelehrbar? die Zuschrift von Gerhard Kühne sowie eine redaktionelle Mitteilung (Der Monat 3 [1951], H. 36, S. 669f.). 
weiterzulesen. Der Bericht des ehemaligen Staatssekretärs Freiherr von Weizsäcker hingegen bemüht sich, einen großen Bogen um dieses schicksalhafte Ereignis zu schlagen, aber selbst hier hilft die absichtliche Entfernung vom Bannkreis nichts; auch hier schlägt die Nadel, fast wider Willen, heftig aus. ${ }^{172}$

An diesem historischen Ereignis, also dem deutschen Widerstand des 20. Juli 1944 gegen den Nationalsozialismus, war es nach dem Dafürhalten von de Mendelssohn unmöglich vorbeizugehen. Und zwar keineswegs aufgrund der politischen Bedeutung, die seiner Meinung nach »beträchtlich« überschätzt wurde, sondern in erster Linie deshalb, »weil in ihm die Kernfrage politischer Moralität enthalten« war. Denn, so der Autor:

Hier scheiden sich in der Tat die Geister, und die Seeoffiziere von den Staatsmännern. Wie sie sich damals im Erlebnis und heute in der Darstellung zu ihm stellen - das ist der Schlüssel. Er ist nicht der einzige, sondern einer unter mehreren, aber er schließt mehr auf, als man glaubt. Es ist nicht nur naheliegend, es ist zwingend, die vorliegende Literatur von dieser Schlüsselstellung aus zu betrachten. ${ }^{173}$

Vor diesem Hintergrund sowie seiner persönlich gemachten Erfahrung wenige Monate nach dem Ende des Zweiten Weltkrieges - im September 1945 in Berlin nahm de Mendelssohn in einem Kino an der Vorführung der Tonfilmaufnahmen vom sogenannten Witzleben-Prozess teil, war und erlebte das »entmenschte Gebrüll des Volksgerichtshofpräsidenten Freisler« und das "schreckerfüllte Murmeln der zusammengeprügelten Angeklagten«, das, so der Autor, die meisten Teilnehmer der Filmvorführung nicht ertragen konnten - setzte die konkrete Auseinandersetzung mit den vier Büchern ein, die sich indes in erster Linie auf das Erinnerungsbuch von Ernst von Weizsäcker bezog. ${ }^{174}$ »Die Witzleben-Gruppe«, so die anfängliche Erläuterung des Autors,

umfaßte, wie erinnerlich, nicht nur eine Reihe hoher Offiziere, sondern auch mehrere frühere Botschafter wie Hassell und Schulenburg und einige verantwortliche Beamte des Ribbentropschen Auswärtigen Amtes. Kaum ein Jahr war damals vergangen seit jenem mörderischen Prozeß, und nun wartete der ehemalige Chef dieser Männer selbst auf seinen eigenen Prozeß im Nürnberger Gefängnis. Das war, so empfand man, in Ordnung. Aber wo war ihr unmittelbarer Vorgesetzter, der ständige Staatssekretär, der das Außenministerium während dieser kritischen Jahre unter Ribbentrop und in seinem Namen geleitet hatte? Weizsäcker war weder hier noch dort. Erwar an drittem Ort -in Rom, wohin er sich gegen Ende des Krieges als Botschafter am Vatikan hatte versetzen lassen. War das in Ordnung?, fragte man sich angesichts dieses Films, in dessen Verlauf sein Name mehrfach erwähnt wurde. Nach der Lektüre seiner >Erinnerungen< ist man nicht mehr so erstaunt, wie man es damals war. ${ }^{175}$

172 De Mendelssohn, Die verhinderten Hochverräter, S. 496.

173 Ebd.

174 Weil sich der Rezensionsaufsatz von de Mendelssohn in erster Linie mit dem Erinnerungsbuch von Ernst von Weizsäcker auseinandersetzte, beziehen sich die nachfolgenden Ausführungen aus Raumgründen fast ausschließlich auf ebenjenes Buch. 
Angesichts der begrifflichen Unterscheidung zwischen "Widerstandsgruppe« und »innere Opposition« war Weizsäcker in den Augen von de Mendelssohn - obwohl diese beiden Gruppen zwischenzeitlich Berührungspunkte hatten - keineswegs Mitglied der Widerstandsgruppe. Es gab für ihn Fälle, »in denen >Opposition< den >Widerstand meidet wie eine ansteckende Krankheit. Der Fall Weizsäcker ist ein solcher « (S. 497). Gleichwohl stand Weizsäcker in »Opposition zum Hitler-Regime«, dessen Ziele er nach de Mendelssohn für »übertrieben hielt (aber auch nicht mehr) und dessen Methoden ihm dilettantisch und daher gefährlich erschienen«. Und zweifelsohne hoffte er, so der Autor, dass irgend jemand sich findet, um »die Verbrecherbande zu beseitigen, ehe es zu spät war und sie Deutschland so ruiniert hatte, daß es keinen verhandlungsfähigen Partner in einem Ausgleichsfrieden mehr abgeben konnte«. Allerdings war Weizsäcker nicht persönlich bereit, »aktiv« zu werden und sich zu beteiligen, um Hitler zu beseitigen. Dazu de Mendelssohn: »Es heißt in den >Erinnerungenর: »[M]an müsse den Sprung wagen, selbst das 75prozentige Risiko hin, daß das Ausland auch dann nicht einlenke [...] der Umsturz müßte freilich so rechtzeitig kommen, daß er nicht zu einer Form der Kapitulation werde.«Und an anderer Stelle:

Ich war selbst nicht der Mann des Attentates [...], ich selbst war nicht eingeschriebenes Mitglied einer solchen Cruppe. Meine ständige Arbeit lag in der außenpolitischen Obstruktion. Meine Ratschläge lauteten seit Spätsommer 1938 nie mehr anders, als daß Hitler zu beseitigen sei.

Die Vorstellung, daß man »eingeschriebenes Mitglied« eines Vereins zur Ermordung von Diktatoren sein könne, ist zu naiv, als daß sie gänzlich naiv sein könnte. ${ }^{176}$

Nach Meinung von de Mendelssohn war der Mitarbeiter von Weizsäcker im Auswärtigen Amt, Dr. Erich Kordt, genauso wenig »eingeschriebenes Mitglied« - trotzdem hinderte es ihn nicht daran, am 1. November 1939 gegenüber General Oster seine Bereitschaft zu erklären, eine »Bombe auf Hitler zu werfen«, weil er zu der Erkenntnis gekommen war, dass die Zeit der »Ratschläge« nämlich vorbei sei. Vor diesem Hintergrund schrieb Kordt in seinem Buch: »Aber Weizsäcker hatte ihn >etwas schärfer angesehen und gesagt: >Ich bedaure, es hat in meiner Erziehung nicht gelegen, einen Menschen zu töten.« De Mendelssohn kommentierte diesen Satz mit folgenden Worten:

Das ist ein ungeheuerlicher Satz. Es fällt rückblickend schwer, zu verstehen, daß diese Bemerkung dem viel jüngeren Kordt nicht die Augen über das menschliche Format seines Vorgesetzten geöffnet hat. In wessen Erziehung lag es denn? Mit derlei verstand der Staatssekretär seinen Mitarbeitern zu imponieren. Dr Kordt bemerkte die monströse Unverschämtheit, die in diesen Worten lag, überhaupt nicht. Er nahm sie für Abgeklärtheit und menschliche Untadeligkeit und akzeptierte bedingungslos eine moralische Autorität, deren Nichtvorhandensein ihm in diesem Augenblick hätte offenbar werden müssen. Welcher Offizier würde es wagen, seinen Soldaten derlei vor dem Sturmangriff zu sagen? Die Episode spricht gegen die Mitarbeiter: sie spricht Bände über das Auswärtige Amt. ${ }^{177}$ 
Für de Mendelssohn war der Staatssekretär des Auswärtigen Amtes, Ernst von Weizsäcker, die exemplarische Verkörperung des »hohen deutschen Staatsbeamten. Ein seltsames, der nichtdeutschen Welt schwer verständliches Wesen« (S. 498), was in seinen Augen auch die Schwierigkeit der ausländischen Staatenwelt, der Alliierten erklärte, die die Schwierigkeit ausmachte, dieses »Phänomen«, dieses »Rätsel« zu verstehen. Mittels der persönlichen Aufzeichnungen versuchte der Autor ebenjenes »Rätsel« $\mathrm{zu}$ fokussieren, zu verstehen und folglich zu erklären.

Von Weizsäcker begann seine Karriere, so de Mendelssohn, als Marineoffizier und ist es, wie es Weizsäcker in seinem Buch selbst betonte, im Grunde genommen immer geblieben. Für de Mendelssohn wiederum haben die sogenannten schweigsamen Berufe der Flotte sowie der Diplomatie in der »deutschen Vorstellung« vieles gemeinsam, sodass Weizsäcker einen Menschentyp verkörperte, der gewissermaßen beiden Berufen zupasskam und der der »Seeoffiziershaltung in der Diplomatie entsprach und der ihm auch den Namen des sgroßen Schweigers eingetragen hatte. "Die unglückliche Vorstellung vom >Staatsschiff«", so die Auffassung von de Mendelssohn, »das wohlbehalten durch alle Stürme manövriert werden muß, führt zu der Vorstellung von der Geschichte als eines Ozeans, in dem Naturgewalten unversehens haushohe Wellen aufwerfen. $^{178}$

Nach dem Urteil von de Mendelssohn koppelten sich in Weizsäcker ebendiese beiden Berufe zu einer »verhängnisvoll irrigen Vorstellung von den Aufgaben der Staatsgeschäfte« (ebd.). In seinen Augen war die Annahme, wenn jemand, der den Mund hält, »unbedingt über etwas schweigt«, ein Irrtum, denn bestand immer auch die Möglichkeit, dass er »überhaupt nichts zu sagen« habe. Und weiter:

Leicht beeindruckbare Untergebene wie der sonst aufgeschlossene und keineswegs unkritische Dr. Kordt halten aber im Fall Weizsäcker just das »nicht für möglich«. Freilich haben gerade die Hitler-Jahre gelehrt, daß politischer Redeschwall ebensosehr Ratlosigkeit und Ideenlosigkeit bemänteln können, und die löbliche Zurückhaltung ist verständlich, um so mehr, wenn Sicherheitserwägungen sie verlangen. Aber der Mythos vom klarblickend-weisen Marineoffizier wurde lange vor Hitler in Deutschland geboren, und Hitler hat ihn ausgenützt. In der Politik aber ist Schweigen keineswegs immer eine Tugend; es kann zu einer schlechten Angewohnheit, sodann zu einem Laster und endlich zum regelrechten Verhängnis werden. »Mißverstanden zu werden«, so schreibt Weizsäcker, »das gehört zu den Risiken des diplomatischen Berufs. «Cewiß; mehr noch aber gehört es zum diplomatischen Beruf, diese Risiken zu vermeiden. »Wer mich nicht von selbst verstand, dem hatte ich nichts mitzuteilen. «Das heißt, die nautische Signalsprache auf die Spitze treiben. Es heißt auch, es sich sehr leicht machen. Zu den Aufgaben des diplomatischen Berufs gehört es in erster Linie, sich verständlich zu machen und verstanden zu werden. ${ }^{179}$ Für de Mendelssohn stand zweifelsfrei fest, dass es für eine oppositionelle bzw. auch nur >bremsende< Diplomatie »in einer wild gewordenen Diktatur« Beschränkungen gab. Gleichwohl konnte seiner Meinung nach »die Tarnung und Ceheimniskrämerei«, die ein totalitäres Regimes seinen Opponenten aufzwang, auch zu weit getrieben werden; bis zu dem Punkt nämlich, wo der Zweck, um dessentwillen man sich tarnt, verloren geht. Hierfür liefert Dr. Kordt ein beredtes Beispiel. Auch 
Weizsäcker erwähnt die geheime Fühlungnahme zwischen dem Auswärtigen Amt und dem britischen Foreign Office zur Zeit der Münchener Krise, aber hier wie an so vielen anderen Stellen schweigt er sich über die Details kryptisch aus. ${ }^{180}$

Angesichts der Erfahrung der letztlich gescheiterten diplomatischen Verhandlungen mit der britischen Regierung im Jahre $1938^{181}$ musste auch Weizsäcker in Gesprächen mit seinen ausländischen »Partnern« erfahren, dass er keine Unterstützung bekam, sodass er sich in seinem Erinnerungsbuch über die "mangelnden hellseherischen Fähigkeiten seiner Partner« beklagte. Hierzu konstatierte de Mendelssohn mit Blick auf die "politische Phantasie« bzw. den »politischen Wirklichkeitssinn« Weizsäckers (sowie auf entsprechende Ausführungen in Kordts Buch):

Die Wahrheit ist, daß die deutsche Opposition einen außerordentlich hohen Vertrauenskredit beanspruchte, ja, daß sie glaubte, ein Recht auf ihn zu haben, ohne die geringsten vorweisbaren Sicherheiten anzugeben. Die Selbstverständlichkeiten, mit der dieser Kredit ausgerechnet im Namen des politischen Urteilsvermögens Baron Weizsäckers verlangt wurde, hat etwas entwaffnend Impertinentes. ${ }^{182}$

Und weiter hieß es hinsichtlich der Klage der »innerdeutschen Opposition«, dass sie infolge der unzureichenden Unterstützung aus dem Ausland an der »Entfaltung behindert « und zudem durch »Gegenzüge jener, die nach ihrer Ansicht mit ihr hätten eines Sinnes sein müssen, paralysiert wurde«, sodass letzten Endes daraus als Ergebnis die "politische Kalamität wie Casablanca und die Forderung nach >Unconditional Surrender« herauskam, dass dies nur die »Hälfte des Argumentes« war. Denn, so de Mendelssohn:

Die andere Hälfte wird von diesen Politikern nicht erkannt und von diesen Memoirenschreibern ignoriert. Sie besteht darin, daß bei den außerdeutschen Hitlergegnern durchweg völlige Unklarheit über Ausmaß, Gewicht und politische Konzeption der innerdeutschen Opposition herrschte. Das war nicht die Schuld des Auslandes. Sowohl

$180 \mathrm{Ebd}$

181 De Mendelssohn beschrieb hier die Verhandlungen der »Brüder Kordt« mit Sir Horace Wilson, der sozusagen als Mittelsmann zu Chamberlain fungieren sollte. Durch dessen Vermittlung kam es daraufhin zu einem Cespräch zwischen Theo Kordt, also dem Bruder von Erich Kordt, und dem britischen Außenminister Halifax, das am 6. September 1938 geführt wurde. Ziel war es, die britische Regierung dazu zu bringen, mit einer »Ceste« die Opposition in Deutschland »zu stützen und ihr für ihre Aktion den nötigen Rückhalt« zu liefern. Dazu schrieb der Autor: »In diesem Gespräch wird Theo Kordt jedoch nie deutlicher als: `die politischen und militärischen Kreise, für die ich spreche und: `die Männer, für die ich spreche«. Kein einziger Name wird auch nur andeutungsweise genannt, der es den Engländern ermöglichen konnte, den Charakter und die Cestalt dieser Opposition abzuschätzen, die sie unterstützen sollten. Die Brüder Kordt waren enttäuscht, als die britische Geste nicht so nachdrücklich ausfiel, wie sie gehofft hatten und wie ihnen nötig erschien; sie verstanden nicht, daß sie etwas [U]nmögliches verlangt hatten: Vertrauen und Unterstützung für Kräfte, die nicht hervorkommen konnten oder wollten und daher dem Unterstützer unbekannte Größen blieben. Die Gründe mögen einleuchten, die Enttäuschung und gar die nachträgliche Klage bleiben dennoch naiv. Eine Zeichensprache, die selbst die Symbole eliminiert und nur schweigend den Partner setwas schärfer ansieht،, funktioniert nicht einmal bei der Marine.« (Ebd., S. 499)

182 Ebd., S. 500. 
Weizsäcker als auch Kordt und Aßmann bestätigen, direkt und indirekt, daß diese Opposition ihren potentiellen Verbündeten und Helfern herzlich wenig Beweise ihrer Existenz geliefert hat. [...] Der 20. Juli 1944 bildete keine vollgültige Ausnahme, denn auch hier ist die politische Vorstellungswelt der Verschwörer bis heute nicht klar hervorgetreten. So schießt das egozentrische deutsche Plädoyer, das Ausland hätte mehr »Verständnis« und »Einfühlungsvermögen« für die komplizierte Lage in Deutschland zeigen müssen, und der ewige Vorwurf Weizsäckers und Kordts, man habe erst Hitler und dann die Opposition »falsch behandelt«, über das psychologisch erreichbare Ziel hinaus und es schließt sich der verhängnisvolle Kreis [...]. ${ }^{183}$

Für den Rezensenten war es keine Überraschung, dass gerade die Engländer für die "Seemannsgewohnheiten« in der Politik, namentlich von Ernst von Weizsäcker, mehr oder weniger ablehnend gegenüberstanden. So schrieb zum Beispiel - wie es de Mendelssohn anführte - der englische Historiker A. J. P. Taylor über den deutschen Staatssekretär in einer Besprechung, angesichts des weizsäckerschen Arguments, dass er, "nachdem der Krieg einmal ausgebrochen war, seinen Posten ebensowenig verlassen konnte wie ein Matrose sein Schiff: 'Es scheint ihm nicht aufgefallen zu sein, daß zwischen den beiden Stellungen ein Unterschied besteht. Andernfalls hätte Hitler das gleiche Argument für sich in Anspruch nehmen können und alle Hauptkriegsverbrecher mit ihm.« De Mendelssohn machte daran anschließend folgende Feststellung:

Hinzuzufügen wäre, [...], daß Staaten nun einmal keine Schiffe sind und die Ceschichte kein Ozean. Weizsäcker, ruhig, besonnen [...] taktvoll, von untadeligen Manieren, war gewohnt, einen leeren Horizont abzusuchen; im dschungelhaften Wirrwarr totalitärer Politik war er verloren. Es liegt nahe anzunehmen, daß Ribbentrop ihn eben aus diesem Grund so lange an der Spitze eines Auswärtigen Amtes beließ, das er längst jeglicher wirklichen Bedeutung entkleidet hatte. ${ }^{184}$

Vor dem Hintergrund der politischen »Karriere« im diplomatischen Dienst der Weimarer Republik - die der »Stuttgarter Ministersohn« im Alter von 37 Jahren begann, nachdem er 20 Jahre lang Dienst in der »kaiserlichen Marine« vollrichtete - schilderte der Autor die Verachtung, mit der Weizsäcker auf die »Amateure« herabblickte, »wie er die sogenannten >Staatsmänner seiner Zeit nennt. >Wir vom Amt< - ist eine Phrase, die von nun $a b$ auf fast jeder Seite seiner Memoiren erscheint.« Diese »Verachtung«, die radikale Abneigung gegen >Parlamentarier<, zeigte sich beispielsweise auch in der negativen Haltung gegenüber dem Völkerbund. ${ }^{185}$ Nicht nur die »Genfer Reden« bereiteten ihm ein Unbehagen, sondern auch die Tatsache, dass die Gewinner der Hauptkonferenz »nach meiner Beobachtung die Vertreter dunkelhaariger Nationen« waren (S. 501). Hierzu und zur so bezeichneten »Erziehung«, auf die sich Weizsäcker berief, sagte de Mendelssohn:

183 Ebd.

184 Ebd.

185 De Mendelssohn konstatierte, dass nach Ansicht Weizsäckers der Völkerbund einerseits »der >deutschen Demokratie den Fangstoß versetzt« hatte und andererseits das Ausland am Aufstieg Hitlers »schuld « war »und sich selbst alles zuzuschreiben hat. Nirgends findet sich ein Wort, daß auch deutsche Kräfte an der erstaunlichen Karriere dieses Mannes beteiligt waren [...].«(Ebd., S. 501) 
Es wird dem Staatssekretär nicht recht sein, des Rassedünkels verdächtigt zu werden. Doch ist an diesem Verdacht das schmallippige Schweigen schuld; er hätte sich deutlicher ausdrücken müssen. Was immer die heimtückisch-herabsetzende Bemerkung über die Vertreter der dunkelhaarigen Nationen bedeuten mag - der Staatssekretär hütet sich, sich zu erklären -, sie ist für die Goebelssche Galerie gemacht, deren Beifall der Staatssekretär sich für bedürftig zu empfinden scheint. Ohne den Seeoffizieren die Ehre abschneiden zu wollen, muß gesagt werden, daß sie aus just jener »Erziehung« stammt, welche Coebbels dann so trefflich zu vulgarisieren verstand. ${ }^{186}$

Im zweiten Teil seines Rezensionsaufsatzes stellte sich der Autor die Frage, die sich aufgrund des bis dahin über von Weizsäcker zur Sprache gebrachten aufdrängte, nämlich:

Wie ist eine solche Persönlichkeit gerüstet, mit der Hitler-Diktatur fertig zu werden? [...] Wie werden »wir vom Amt unter den neuen Herren fahren? Weizsäcker, als Aristokrat, Monarchist und `Professionak, hegt keine Sympathien für Hitler. Er wittert den >Amateur und fürchtet für seinen guten Namen. Aber er blickt dem Problem nicht gerade ins Cesicht«(S. 502).

Für de Mendelssohn ging es Weizsäcker - als auch Kordt - darum, den Apparat des Auswärtigen Amtes zu erhalten, der nicht blind den Befehlen des "Nazi-Politbüros « ${ }^{187}$ gehorcht: und zwar um sich nicht selbst auszuschalten und sich damit einen Einfluss auf die weitere Entwicklung zu erhalten. Um diesen Zweck zu erreichen, blieben Weizsäcker (sowie Kordt) im Amt und hielten bis zum Schluss aus. Nach Auffassung von de Mendelssohn war dies die zentrale Entscheidung. Alles andere folgte aus ihr. Beide blieben, um >das Schlimmste zu verhüten<, zum Zweck der saußenpolitischen Obstruktion<.

Für ihn hatten allerdings Weizsäcker (sowie Kordt) letzten Endes nichts erreicht. Und in diesem Zusammenhang stellte er fest, dass durchaus die Möglichkeit bestand, dass das Verbleiben im Amt nicht nur das »Üble nicht besonders behindert und gestört, sondern ihm sogar Vorschub geleistet, Deckung verschafft, wider Willen und Absicht Prestige geliehen hat «. Insbesondere dieser Aspekt war in seinen Augen von Anbeginn das Problem in dem totalitären Staat, in dem "gemäßigte, neutrale, bremsende Elemente noch Schlüsselstellungen - oder was von außen wie eine Schlüsselstellung aussieht - inne[hatten]«. Und daraus ergaben sich für de Mendelssohn die Fragen: »Ist der Versuch auf alle Fälle gerechtfertigt oder nur durch den Erfolg? Ist der Mißerfolg der schlüssige Beweis dafür, daß die Methode falsch, gefährlich, ja verderblich war? ${ }^{188}$

Weizsäcker berief sich immer wieder auf Prinzipien, die er noch »verantworten« könne, so der Rezensent, wenn es um das Ausführen von »Weisungen aus Berlin« ging.

186 Ebd.

187 Anspielung von de Mendelssohn auf eine-gewissermaßen totalitarismustheoretische-»Analogie« von Dr. Kordt, der in seinem Memoirenband das Verhalten »russische[r] Beamtenschaft bei der bolschewistischen Revolution « ansprach und in diesem Zusammenhang zu der Auffassung gelangte, dass die »bolschewistische Regierung« einen Apparat geschaffen hat, »der den Befehlen des Politbüros blind gehorchte (ebd., S. 502). 
Das zentrale Problem bestand jedoch unter anderem darin, dass der Staatssekretär keine eigentlichen Prinzipien hatte, sodass er - quasi als exemplarische Figur - von der nationalsozialistischen »Revolution« nach dem »Blutbad des 30. Juni 1934« als "schwankender und halbentschlossener Diener" Schritt um Schritt korrumpiert wurde (S. 502). Und die oftmals an sich selbst gestellte Frage, ob er zurücktreten oder im Amt bleiben solle, wurde immerfort von Weizsäcker zugunsten des Verbleibens beantwortet, und zwar mit dem Argument, dass der »Fachmann« dem »gefährlichen Dilettanten seinen Platz nicht räumen« dürfe (S. 503). Dazu der Kommentar von de Mendelssohn:

Da sind sie wieder, Fachmann und Dilettant; die Dilettanten haben gewechselt, der Fachmann ist der gleiche. Man muß sich fragen, was mit diesen beiden Begriffen, die in allen diesen Büchern eine große Rolle spielen, in diesen Zusammenhängen überhaupt anzufangen ist. Zweifellos wird mit ihnen ein beträchtlicher Unfug getrieben. Wer war denn hier, wer ist denn auch heute in der Politik der Fachmann und wer der Dilettant? [...] Für Hitler und Ribbentrop waren Weizsäcker und Kordt Dilettanten, wenn auch sehr gefährliche. [...] Politik braucht man nicht »gelernt« zu haben wie die Zahntechnik; es sind nicht selten die gelernten Kräfte, die das Unheil anrichten, wenn die Dinge sich nicht nach dem Leitfaden entwickeln. Niemand hatte eine bessere Gelegenheit, gerade dies zu erkennen und diese Erkenntnis zu verwerten als Weizsäcker. Gerade er bleibt ihr gegenüber völlig blind und versucht, ein Rudel Wölfe mit Kompaß und Sextant zu dirigieren, als wäre es ein Panzerkreuzer.

In diesem Zusammenhang kam de Mendelssohn in Anbetracht der Rolle Weizsäckers in der nationalsozialistischen Außenpolitik bzw. im Auswärtigen Amt im Allgemeinen und in seinem oben angesprochenen Selbstverständnis gegenüber Hitler und Ribbentrop im Besonderen zu folgender Einschätzung:

Vorsicht ist also geboten, auch wenn man Weizsäckers orthodoxe und unwirkliche Konzeption um des Argumentes willen akzeptiert. Denn hier handelt es sich noch um etwas anderes. Wie wird hier argumentiert? Warum durfte der Fachmann dem gefährlichen Dilettanten den Platz nicht räumen? Es wird vorausgesetzt, daß der Fachmann ein Patriot ist, dem das Wohlergehen der Nation vor allem am Herzen liegt. Ohne des Fachmanns Hilfe muß der Dilettant mit seinen Methoden Schiffbruch erleiden und die Nation ins Unglück stürzen. Das kann der Fachmann nicht dulden, weder als Fachmann noch als Patriot. Er muß deshalb dem Dilettanten und der Nation beispringen und helfen, ob die Hilfe nun erwünscht ist oder nicht. ${ }^{189}$

Im nächsten Abschnitt seiner Rezension des Buches von Weizsäcker kam de Mendelssohn auf den Nürnberger Prozess ${ }^{190}$ zu sprechen, den das ehemalige Mitglied der NSDAP und der SS in seinem Erinnerungsbuch sozusagen behandeln musste (da er im sogenannten Wilhelmstraßen-Prozess Hauptangeklagter war). Da Weizsäcker wenig mehr als eine Rekapitulation der vor dem Gericht vorgebrachten Argumente lieferte,

189 Ebd., S. 503.

190 Hier setzte der Autor, wie er anmerkte, das Wissen des Lesers des Monat voraus, sodass er keine weiteren Erläuterungen vornahm. 
stellte »das Buch eine Rechtfertigungsschrift« dar (S. 504). Im oben angesprochenen Zusammenhang mit der "Suche nach dem geringeren Übel« zeichneten sich für de Mendelssohn hinsichtlich der Genesis des Zweiten Weltkrieges zwei Hauptphasen ab. Zum einen die Phase bis zum Ausbruch des Krieges, wo es das Ziel war, ebendiesen Ausbruch zu verhindern, »obwohl dies die Beibehaltung des Hitlerregimes bedeuten« musste: Hitler war das "geringere Übel«. Und zum anderen die Phase vom Beginn des Zweiten Weltkrieges, wo es Weizsäckers Ziel war, den Frieden auf verschiedene Weise "zurückzubringen « und somit den Krieg abzukürzen, selbst wenn dies die »Beseitigung des Hitlerregimes durch eine Gewaltmaßnahme bedeuten « musste: Hierzu hieß es bei de Mendelssohn: "Der Staatsstreich ist das kleinere Übel, der völlige Zusammenbruch das größere. Auch hier wünschte man sich, Weizsäcker hätte an irgendeiner Stelle diese Alternativen einmal klipp und klar dargelegt. Man muß es für ihn tun. ${ }^{191}$

Vor diesem Hintergrund stellten sich für de Mendelssohn zwei Fragen. Ob nämlich das Auswärtige Amt und die Stellung von Weizsäckers ihm zu irgendeinem Zeitpunkt die Möglichkeit in die Hand gab, »den Ausbruch des Krieges zu verhindern oder auch nur zu verzögern«? Dies wurde vom Staatssekretär verneint, der die eigene Machtlosigkeit einräumte - und diese Ansicht vertrat auch de Mendelssohn. Gleichwohl, obwohl Weizsäcker (sowie Kordt) sich permanent »der Unzulänglichkeit und Ungeeignetheit ihres Apparates völlig bewußt« waren, fuhren sie fort. Dazu de Mendelssohn:

Sie werden Opfer ihrer Illusionen, nachdem sie ihre Illusionen geopfert haben. Gerade das aber sollte Fachleuten nicht passieren. In diesem Licht besehen, gewinnen Professor Taylors Vorwürfe mehr als rein polemisches Gewicht.Anfänglich versucht Weizsäcker, sich Ribbentrop zu bedienen und durch ihn zu arbeiten. [...] Als sich herausstellt, daß er nichts erreicht, versucht er, gegen Ribbentrop und Hitler zu arbeiten, indem er allzu schweigsam - mit ausländischen Diplomaten konspiriert. Aber bei dem einen, einzigen wirklich ernsten Versuch, Hitler in die Zügel zu fallen, wird die delikate Aufgabe schließlich den Brüdern Kordt überlassen. ${ }^{192}$

Vor dem Hintergrund der Erinnerungen des Panzergenerals Heinz Guderian ${ }^{193}$ war für de Mendelssohn - der mit Blick auf die »Fragen der politischen Moralität« hier keineswegs den >dummen Generak gegen den >klugen Diplomaten« gegeneinander ausspielen wollte - die Möglichkeit des Zurücktretens keineswegs so kompliziert, wie es Weizsäcker in seinem Buch »nachträglich weismachen« wollte. Sowohl vor als auch unmittelbar nach Kriegsausbruch bestand sehr wohl die Möglichkeit, wie es Weizsäcker selber einräumte, seine Entlassung bei Ribbentrop durchzusetzen, wenn er es wirklich gewollt hätte. »Aber«, so de Mendelssohn:

191 Ebd., S. 504.

192 Ebd., S. $504 \mathrm{f}$.

193 Der Autor zitierte hier aus dem Buch Heinz Guderian, Erinnerungen eines Soldaten, Heidelberg 1950, folgende Stelle: „Wer andere[r] Ansicht war als Hitler, hatte die Pflicht, ihm das offen zu sagen, wenn nicht im Inland, dann aus dem Ausland. Dies gilt in erster Linie und ganz besonders für die Zeit, als es noch Zweck hatte, nämlich für die Zeit vor dem Kriege. Ich muß ablehnen, jene heute Widerstandskämpfer zu nennen, die nur hinter den Kulissen getuschelt haben, daß sie anderer Ansicht seien, die nur andere Leute anzustiften versuchten« (ebd., S. 505). 
er gibt auch zu, daß er nicht gewollt hat. Er gesteht ein, daß das Auswärtige Amt jetzt jeden Anschein eines tatsächlichen Wirkungskreises eingebüßt hat, und es wird unverständlich, wie er glauben konnte, in seinem Rahmen die zweite Hälfte der »selbsterwählten«Aufgabe zu erfüllen - nämlich die Abkürzung des Krieges und Wiederbeschaffung des Friedens -, um derentwillen er es ablehnt, seine Entlassung zu forcieren, im Amt bleibt und ein neuerliches Odium auf sich nimmt.

»lch wollte ihn (den Abschied) nicht erzwingen. Je mehr man in die Hitler-Küche hineinroch, um so stärker fühlte man die Pflicht, dazubleiben und die Giftmischerei zu verhindern.«

Doch verhinderte man die Mischerei eines einzigen Giftes? Kannte man überhaupt die Rezepte? Und ist man nicht beim häufigen Kosten selbst ein bißchen mitvergiftet worden? ${ }^{194}$

Weizsäcker verblieb auch nach dem deutschen Überfall auf Polen und die Sowjetunion durch die Wehrmacht im Amt und alle realitätsfernen und - so könnte man sagen unpolitischen Friedensbemühungen - so wie er sie verstand - blieben erfolglos. Insbesondere auch deshalb, weil er nach Meinung von de Mendelssohn gar nicht erkannte, dass »Hitler den Krieg von Anfang an als ein Mittel zum Zweck seiner Verewigung und Unabsetzbarkeit ansah" und sich Weizsäcker im Grunde genommen in seinem Erinnerungsbuch schon gar nicht mehr daran erinnerte, »wer den Krieg eigentlich angefangen«hatte (S. 505 f.). Und nachdem sein ehemaliger Vorgesetzter, von Ribbentrop, von den Alliierten im Nürnberger Hauptkriegsverbrecherprozess zum Tode durch den Galgen verurteilt wurde, fragte sich Weizsäcker, angesichts der Tatsache, dass er im Wilhelmstraßen-Prozess verurteilt wurde, in einer »merkwürdigen Umkehrung«: »Wie könnte man mir einen Prozeß dafür machen, daß ich, um den Krieg zu vermeiden und ihn abzukürzen, im Amt geblieben war? Ich hätte es eher einer Hitler-Staatsanwaltschaft gegönnt, mich vor einen Volksgerichtshof zu ziehen - denn dort hätten sie und ich in der richtigen Front gestanden.«

De Mendelssohn kommentierte diese Passage mit folgenden Worten:

Es ist unmöglich, an die Aufrichtigkeit dieses Satzes zu glauben. Wenn dies Stolz und Courage sind, dann sind es Stolz und Courage im nachhinein, und sie kommen von einem Mann, der sein Äußerstes tat, um dem Volksgerichtshof aus dem Weg zu gehen, und noch in Rom als Botschafter beim Vatikan zitterte, er könne doch in den Prozeß des 20. Juli hineingezerrt werden. ${ }^{195}$

Nach dem Dafürhalten von de Mendelssohn hätte Weizsäcker im Unterschied zu »Pater Wehrle«, der im bereits erwähnten Witzleben-Prozess als Mitangeklagter des »20. Juli« eine »unvergeßlich eindrucksvolle moralische Figur« abgab - für Wehrle gab es so etwas wie das >geringere Übek nicht, konnte es gar »nicht geben« - aller Voraussicht nach auch nicht »annähernd« eine so moralisch integere Figur abgegeben. »In Wahrheit spielte sich der Kampf Weizsäckers«, so de Mendelssohn weiter, 
der von der Geschmacklosigkeit des oben zitierten Satzes gekrönt wird, auf einem Gebiet ab, das von der Front und ihren Schützengräben sehr weit entfernt lag. Das »Amt«, welches Weizsäcker repräsentierte, und der Menschentyp, der er war, hatten aufgehört, von Cewicht zu sein und spielten längst keine Rolle mehr. [...] Die Maschinerie, die er zu betreuen gewohnt war, war längst zum alten Eisen geworfen worden, und das neue Monstrum, welches mit Volldampf über die Erde raste, verlangte einen anderen Typ von Mechaniker. Genau genommen, es verlangte einen Mann, der entschlossen und imstande war, einen wirklich großen Schraubenschlüssel ins Cetriebe zu werfen. Weizsäcker war nicht dieser Mann, und er besaß keinen Schraubenschlüssel. Hitler und Ribbentrop wußten das und hatten nichts dagegen, daß er auch weiterhin herumstand und den Eindruck erweckte, als sei er der Maschinist. Unter diesen Umständen rangierte er geschäftig seine kleine Lokomotive ohne Zug dahinter auf dem Abstellgleis hin und her, während die großen Höllenexpresse auf dem Hauptgleis dröhnend an ihm vorbeidonnerten. ${ }^{196}$

Darüber hinaus stellte sich für de Mendelssohn in diesem Zusammenhang die für ihn zentrale Frage, ob nämlich der Zweck die Mittel rechtfertigte? Genauer: »Rechtfertigte das den Weg, selbst wenn sich voraussehen ließ oder doch auf halbem Weg herausstellen mußte, daß am Ende des Weges gar kein Ziel vorhanden war? « Und angesichts von Ausführungen Weizsäckers in seinem Buch - wo dieser unter anderem die Frage aufwarf: ,Warum ich bei völligem innerem Widerstand gegen die herrschenden Menschen, Maximen, Methoden und Motive meinen Namen zu einer fast aussichtslosen Arbeit hergegeben hatte, das mußte ich mit mir selbst abmachen.く - äußerte sich de Mendelssohn folgendermaßen:

Man wird die Berechtigung dieses Anspruchs bezweifeln dürfen. Es ist höchst fraglich, ob ein Mann in der Stellung Weizsäckers seine Taten und Unterlassungen der öffentlichen Beurteilung kurzerhand entziehen und »mit sich selbst abmachen« kann. Diese Formel ist eine bequeme Hintertür, durch die man versuchen kann hinauszuschlüpfen, aber sie bleibt eine Hintertür. Es trifft vermutlich zu, daß die Politik nur allzu häufig auf nicht mehr hinausläuft als eine Suche nach dem geringeren Übel. Aber das Entscheidende ist doch wohl, daß sie es nicht sollte und daß es niemals vorgefaßtes Ziel sein darf. Wenn man die Suche nach dem geringeren Übel überhaupt als politische Zielsetzung zugestehen will, dann doch nur in der Voraussetzung, daß sie an dem Crad ihres Erfolges bemessen wird. Nur der Erfolg kann diese Zielsetzung rechtfertigen, und hier, wenn überhaupt irgendwo, spricht die Erfolglosigkeit das Urteil. Weizsäcker freilich fand das geringere Übel nicht, aus dem einfachen Grund, weil es nicht vorhanden war, es sei denn, daß er sich selbst als das geringere Übel ansah. Man kann sich des Gefühls nicht erwehren, daß ihm daran liegt, beim Leser diesen Eindruck zu erwecken: man hätte uns machen lassen sollen, und diese Sache wäre nicht so schlimm geworden!197

Angesichts des »lückenhaft-hochfahrende[n] Bericht[s] des schweigsamen Staatssekretärs« (S. 507) und insbesondere aufgrund von Weizsäckers Vorstellung, dass er die zurückliegende deutsche Geschichte, genauer gesagt, das zurückliegende »Schicksal

196 Ebd., S. $506 f$.

197 Ebd., S. 507. 
seines Volkes, Europas, der Welt« mit `sich selbst abmachen` zu können glaubte, kam de Mendelssohn zum Abschluss seiner Auseinandersetzung mit den »Erinnerungen« von Ernst von Weizsäcker in seinem Rezensionsaufsatz Die verhinderten Hochverräter ${ }^{198}$ zu der Ansicht, dass genau dies nicht möglich sei und er darauf keinen Anspruch hat. »Nichts anderes«, so de Mendelssohn weiter,

sagten schließlich auch die Hauptkriegsverbrecher in Nürnberg in ihren Schlussworten: daß sie für das, was sie getan hätten, nur vor sich selbst, vor ihrem Gewissen oder bestenfalls vor dem deutschen Volk sich zu verantworten hätten - nicht aber vor der Welt, die sie für Tat und Untat in Schutt und Asche gelegt hatten. Man drehe es, wie man will. Mit diesem einen Satz, wenn schon mit nichts sonst in seiner Rechtfertigungsschrift, hat Weizsäcker die moralische Rechtfertigung - und sie ist hier die politische! - für seinen Prozeß selbst geliefert. ${ }^{199}$

\subsection{Die nationalsozialistischen Massenverbrechen und die "Endlösung der Judenfrage"}

Nach den frühzeitigen Hinweisen im ersten Heft des Monat auf einzelne Tagebucheintragungen von Goebbels aufgrund von dessen persönlichen ideologischen Vorstellungen, Zielen und Kommentaren in Bezug auf den nationalsozialistischen Antisemitismus und die »Judenpolitik« des NS-Systems ${ }^{200}$ war es Lasky, der bereits zwei Ausgaben später in der bereits angesprochenen Rezension des Buches Vom anderen Deutschland. Aus den nachgelassenen Tagebüchern 1938 bis 1944 auf Eintragungen Ulrich von Hassells aufmerksam machte, in denen dieser auf Massenverbrechen des NS-Systems einging. Von Hassell berichtete nämlich unter anderem von »Massentötungen« polnischer Juden durch die SS nach dem Überfall auf Polen, »die den deutschen Namen befleckt haben«. Des Weiteren zitierte Lasky Eintragungen, in denen zum Ausdruck kam, dass von Hassell erschüttert war angesichts der »unaussprechliche[n] Judenmorde« durch die SS, die mit ihren Maschinenpistolen Kinder in einem Ghetto töteten, die sich »un-

198 Zur »Cewissensfrage«, die Ernst von Weizsäcker, Erich Kordt und Vizeadmiral a. D. Kurt Aßmann in ihren Büchern zur Sprache brachten, schrieb de Mendelssohn u. a.: »[W]arum die deutsche Opposition unter den gegebenen Umständen nicht die letzte logische Konsequenz gezogen hat und bis zum militärischen Verrat gegangen ist, um Hitlers Niederlage zu beschleunigen« (ebd., S. 508f.). Mit Blick auf die Position von Admiral Aßmann, der dem »Verrat«ablehnend gegenüberstand, stellte de Mendelssohn für alle drei genannten Personen fest, »daß es bei aller Hitler-Gegnerschaft patriotische Pflicht war, trotzdem das Äußerste zu tun, um selbst in diesem ungerechtesten aller Kriege den Sieg zu erringen, wenn es nur irgend ging «(S. 508). Und weiter: »daß es auch für die Hitler-Gegner innerhalb Deutschlands einen sglücklichen Ausgang des Krieges im Sinne Hitlers gab, daß ihnen der >Endsieg`noch dringender am Herzen lag als der Wunsch, Hitler `abzuschüttel[n]<, wie Weizsäcker es ausdrückt. <Gibt das nicht jenen recht, die außerhalb Deutschlands geltend machten, so lange Hitler siegte, habe man in Deutschland im Grund gegen ihn nichts einzuwenden, nur einen sverlierenden Hitler müsse man natürlich sabschütteln«? Wird hier nicht der Primat der politischen Moral doch letzten Endes dem militärischen Erfolg untergeordnet?« (S. 509).

199 Ebd., S. 508.

200 Siehe im Einzelnen oben das Kap. IV.1.3. 